\title{
The signature of Higgs boson near surface horizon of oscillating cylindrical Universe
}

\author{
Alireza Sepehri ${ }^{1}$, Somayyeh Shoorvazi ${ }^{2}$ \\ ${ }^{1}$ Faculty of Physics, Shahid Bahonar University, Kerman, Iran \\ ${ }^{2}$ Islamic Azad University, Neyshabur branch, Neyshabur, Iran
}

Email address:

A.Sepehri14@gmail.com (A. Sepehri), S.Shoorvazi14@gmail.com (S. Shoorvazi)

To cite this article:

Alireza Sepehri, Somayyeh Shoorvazi. The Signature of Higgs Boson Near Surface Horizon of Oscillating Cylindrical Universe, American Journal of Modern Physics. Vol. 2, No. 3, 2013, pp. 124-131. doi: 10.11648/j.ajmp.20130203.15

\begin{abstract}
First, by using cylindrically symmetric model, the quantum state for cyclic Universe is studied. It is shown that the ground state for oscillating cylindrical Universe is a maximally entangled with two-mode squeezed states on expanding and contracting Hilbert spaces of Universe. Next, the production cross section for each Universe state is obtained. It is observed that the probability for producing each state in cyclic Universe depends on different oscillating frequencies of Universe in space-time directions.After that, the effect of Universe oscillation on Higgs boson cross section is considered. To calculate the production cross section for Higgs boson, the production cross section for each Universe state is multiplied by thermal distribution of Higgs boson .It is found that in each Universe state, different amount of Higgs boson is produced. Finally, the signature of Higgs boson near surface horizon of oscillating Universe is studied. When Higgs bosons produce, they decay to quarks and gluons. Thus an enhancement of these partons can be a signature of Higgs boson inside the cyclic Universe.
\end{abstract}

Keywords: Cyclic Universe, Cylindrically Symmetric Model, Higgs Boson

\section{Introduction}

In recent years, there has been considerable interest in cylindrically symmetric model[1-11] for Universe because this model plays an important role in the study of the heavy particles like Higgs bosons in the early universe. These particles produce during the phase transition after the big bang explosion as the temperature goes down below some critical temperature. Then they decay to QCD matters $[12,13]$.Since the temperature of early universe is very high, we expect more particles to be produced from Higgs bosons. In fact there can be an enormous amount of quark and gluon production from Higgs boson, much more than expected from normal pQCD processes[14,15]. The present question is the possibility of solving the Big-Bang singularity and coincidence problem in oscillating cylindrically symmetric model for Universe?

The idea of an oscillating universe was first proposed by Tolman in the 1930's. There have been many discussions on such a topic and a number of models have been proposed[16], among which there are cyclic models in DGP braneworld scenario, non singular cosmology [17-19], Cosmology without inflation [20], cyclic cosmologies with propagating Lorentz connection modes of spin zero [21] and so on. These proposals present a very broad conceptual framework, in which the big bang is not the beginning of time, but rather a physical event amenable to a testable physical description. In these models, the universe oscillates through a series of expansions and contractions. The quantum entanglement between these expanding and contracting branches of the universe might have observable consequences in the dynamical and thermodynamical properties of one single branch, making therefore testable the whole oscillating universe proposal, at least in principle. The aim of this paper is to consider the effect of Universe oscillation on Higgs boson production cross section.

The outline of the paper is as the following. In section II we study the Higgs boson equation near Universe surface horizon in cyclic cosmology .In section III, we obtain the thermal distribution for different states of oscillating cylindrical Universe. In section IV we consider the Unruh states for Higgs boson near oscillating surface horizon and finally, we analyze the signature of Higgs boson inside the cyclic cylindrical Universe in section V. 


\section{Higgs Boson Near Surface Horizon of Oscillating Universe}

In this section, we write Higgs boson equation inside the oscillating cylindrically symmetric Universe which is more complete than spherically symmetric Universe. To explain cylindrically symmetric Universes, we use of cylindrical coordinate [1-11] we should solve this equation in non flat space-time due to acceleration of Universe. For this reason, we introduce reparameterizations and get Universe metric. We show that acceleration of Universe causes to formation of one surface horizon outside of Universe boundary surface.

The Higgs boson equation in cylindrical coordinate is as $[1,12,13]$ :

$$
\left\{-\frac{\partial^{2}}{\partial t^{2}}+\frac{\partial^{2}}{\partial \rho^{2}}+\frac{\partial^{2}}{\partial z^{2}}+\frac{\partial^{2}}{\rho^{2} \partial \theta^{2}}\right\} \text { Higgs boson }=0(1)
$$

where we have used of this fact that $\mathrm{c}=1$ in national unit. After following reparameterizations:

$$
\begin{aligned}
\rho & \rightarrow R(\rho, z, \theta, t) \\
z & \rightarrow X(\rho, z, \theta, t) \\
\theta & \rightarrow \beta(\rho, z, \theta, t) \\
t & \rightarrow \tau(\rho, z, \theta, t)
\end{aligned}
$$

The Higgs boson equation should be written as:

$$
\begin{aligned}
& {\left[\left\{\left(\frac{\partial R}{\partial z}\right)^{2}+\left(\frac{\partial R}{\partial \rho}\right)^{2}+\left(\frac{\partial R}{\partial \theta}\right)^{2}-\left(\frac{\partial R}{\partial t}\right)^{2}\right\} \frac{\partial^{2}}{\partial R^{2}}+\right.} \\
& \left\{\left(\frac{\partial X}{\partial z}\right)^{2}+\left(\frac{\partial X}{\partial \rho}\right)^{2}+\left(\frac{\partial X}{\partial \theta}\right)^{2}-\left(\frac{\partial X}{\partial t}\right)^{2}\right\} \frac{\partial^{2}}{\partial X^{2}}+ \\
& \frac{1}{\rho^{2}}\left\{\left(\frac{\partial \beta}{\partial z}\right)^{2}+\left(\frac{\partial \beta}{\partial \rho}\right)^{2}+\left(\frac{\partial \beta}{\partial \theta}\right)^{2}-\left(\frac{\partial \beta}{\partial t}\right)^{2}\right\} \frac{\partial^{2}}{\partial \beta^{2}}+ \\
& \left.\left\{\left(\frac{\partial \tau}{\partial z}\right)^{2}+\left(\frac{\partial \tau}{\partial \rho}\right)^{2}+\left(\frac{\partial \tau}{\partial \theta}\right)-\left(\frac{\partial \tau}{\partial t}\right)^{2}\right\} \frac{\partial^{2}}{\partial \tau^{2}}\right] \text { Higgs boson }=0
\end{aligned}
$$

We can set boundary surface of Universe at 1 , by choosing following choices for $\mathrm{R}, \mathrm{X} \beta$ and $\tau$ :

$$
\begin{aligned}
& R(\rho, z, \theta, t)=\frac{\rho}{L(\rho, z, \theta, t)}, \\
& X(\rho, z, \theta, t)=\frac{z}{L(\rho, z, \theta, t)}, \\
& \beta(\rho, z, \theta, t)=\frac{\theta}{L(\rho, z, \theta, t)} \\
& \tau(\rho, z, \theta, t)=\frac{t}{L(\rho, z, \theta, t)}
\end{aligned}
$$

where $L(\rho, z, \theta, t)$ is the Universe boundary surface equation. With above consideration, the Higgs boson equation is written as:

$$
(-g)^{1 / 2} \frac{\partial}{\partial x^{\mu}}\left[g^{\mu v}(-g)^{1 / 2} \frac{\partial}{\partial x^{v}}\right] \text { Higgs boson }=0
$$

where $x^{0}=\tau, x^{1}=R, x^{2}=X, x^{3}=\beta$ and the metric elements are obtained as:

$$
\begin{aligned}
& g^{11}=(R)^{2}\left\{\frac{L_{X L}^{2}+L_{\beta L}^{2}-\dot{L}^{2}}{L^{2}}\right\}+\frac{\left(L-R L L_{R L}\right)^{2}}{L^{4}} \\
& g^{22}=(X)^{2}\left\{\frac{L_{R L}^{2}+L_{\beta L}^{2}-\dot{L}^{2}}{L^{2}}\right\}+\frac{\left(L-X L L_{X L}\right)^{2}}{L^{4}} \\
& g^{33}=(\beta)^{2}\left\{\frac{L_{R L}^{2}+L_{X L}^{2}-\dot{L}^{2}}{L^{2}}\right\}+\frac{\left(L-\beta L L_{\beta L}\right)^{2}}{L^{4}} \\
& g^{00}=(\tau)^{2}\left\{\frac{L_{R L}^{2}+L_{\beta L}^{2}+L_{X L}^{2}}{L^{2}}\right\}+\frac{(L-\tau L \dot{L})^{2}}{L^{4}} \\
& g^{21}=\frac{\dot{L}^{2}}{L^{2}} R X-\frac{L_{X L} R}{L^{3}}\left(L-X L L_{X L}\right)-\frac{L_{R L} X}{L^{3}}\left(L-R L L_{R L}\right) \\
& g^{31}=\frac{\dot{L}^{2}}{L^{2}} R \beta-\frac{L_{\beta L} R}{L^{3}}\left(L-\beta L L_{\beta L}\right)-\frac{L_{R L} \beta}{L^{3}}\left(L-R L L_{R L}\right) \\
& g^{32}=\frac{\dot{L}^{2}}{L^{2}} X \beta-\frac{L_{\beta L} X}{L^{3}}\left(L-\beta L L_{\beta L}\right)-\frac{L_{X L} \beta}{L^{3}}\left(L-X L L_{X L}\right) \\
& g^{10}=-\frac{\dot{L}}{L} R\left(\frac{\partial \tau}{\partial t}\right)-\frac{L_{X L}}{L} R\left(\frac{\partial \tau}{\partial z}\right)-\frac{L_{\beta L}}{L} R\left(\frac{\partial \tau}{\partial \theta}\right)+\left(\frac{1-L_{R L} R}{L}\right)\left(\frac{\partial \tau}{\partial \rho}\right) \\
& g^{20}=-\frac{\dot{L}}{L} X\left(\frac{\partial \tau}{\partial t}\right)-\frac{L_{R L} X}{L}\left(\frac{\partial \tau}{\partial \rho}\right)-\frac{L_{\beta L} X}{L}\left(\frac{\partial \tau}{\partial \theta}\right)+\left(\frac{1-L_{X L} X}{L}\right)\left(\frac{\partial \tau}{\partial z}\right) \\
& g^{30}=-\frac{\dot{L}}{L} \beta\left(\frac{\partial \tau}{\partial t}\right)-\frac{L_{R L} \beta}{L}\left(\frac{\partial \tau}{\partial \rho}\right)-\frac{L_{X L} \beta}{L}\left(\frac{\partial \tau}{\partial z}\right)+\left(\frac{1-L_{\beta L} \beta}{L}\right)\left(\frac{\partial \tau}{\partial \theta}\right)
\end{aligned}
$$

where we have defined:

$$
L_{X L}=\frac{\partial L}{\partial z}, L_{R L}=\frac{\partial L}{\partial \rho}, L_{\beta L}=\frac{\partial L}{\partial \theta}, \dot{L}=\frac{\partial L}{\partial t}
$$

After some mathematical manipulations, the surface coordinate of Universe horizon can be calculated as:

$$
\begin{gathered}
\rho_{\text {horizon }}=R L=L \frac{L_{R L} \pm \sqrt{\dot{L}^{2}-L_{X L}^{2}-L_{\beta L}^{2}}}{L_{R L}^{2}+L_{X L}^{2}+L_{\beta L}^{2}-\dot{L}^{2}} \\
z_{\text {horizon }}=X L=L \frac{L_{X L} \pm \sqrt{\dot{L}^{2}-L_{R L}^{2}-L_{\beta L}^{2}}}{L_{R L}^{2}+L_{X L}^{2}+L_{\beta L}^{2}-\dot{L}^{2}} \\
\theta_{\text {horizon }}=\beta L=L \frac{L_{\beta L} \pm \sqrt{\dot{L}^{2}-L_{R L}^{2}-L_{X L}^{2}}}{L_{R L}^{2}+L_{X L}^{2}+L_{\beta L}^{2}-\dot{L}^{2}} \\
t_{\text {horizon }}=\tau L=L \frac{\dot{L} \pm \sqrt{L_{R L}^{2}+L_{X L}^{2}+L_{\beta L}^{2}}}{L_{R L}^{2}+L_{X L}^{2}+L_{\beta L}^{2}-\dot{L}^{2}}
\end{gathered}
$$

If Universe has the different acceleration in the $\mathrm{z}, \mathrm{t}, \boldsymbol{\theta}$ and ${ }^{\rho}$ directions, Universe has cylindrical symmetry; however, spherical Universes have the same accelerations in these directions. Also, if $\rho_{\text {horizon }}<L(0, z, \theta, t), z_{\text {horizon }}<L(\rho, 0, \theta, t)$, $t_{\text {horizon }}<L(\rho, z, \theta, 0)$ and $\theta_{\text {horizon }}<L(\rho, z, 0, t)$ the surface horizon divides the Universe into two spaces.Particles that are created in one space don't achieve to particles in another space.

\section{The Squeezed States of Oscillating Cylindrical Universe}

In this section, the squeezed coherent states of four dimensional oscillating Universe are explored. An oscillating 
universe is a non-standard scenario of cosmology which, by having the universe expanding and contracting alternately, is expected to solve the Big-Bang singularity and coincidence problem. In this scenario, the surface coordinates of Universe horizon, should be satisfied in following equations[16-21]:

$$
\begin{gathered}
\ddot{\rho}_{\text {horizon }}+\omega_{\rho}^{2} \rho_{\text {horizon }}=0 \\
\ddot{z}_{\text {horizon }}+\omega_{z}^{2} z_{\text {horizon }}=0 \\
\ddot{\theta}_{\text {horizon }}+\omega_{\theta}^{2} \theta_{\text {horizon }}=0 \\
\ddot{t}_{\text {horizon }}+\omega_{t}^{2} t_{\text {horizon }}=0
\end{gathered}
$$

where $\omega_{\rho}, \omega_{z}, \omega_{\theta}$ and $\omega_{t}$ are oscillating frequencies of cylindrical Universe. The horizon coordinate opera$\operatorname{tors}\left(\hat{\rho}_{\text {horizon }}, \widehat{z}_{\text {horizon }}, \hat{\theta}_{\text {horizon }}\right.$ and $\left.\widehat{t}_{\text {horizon }}\right)$ and related mo$\operatorname{mentums}\left(\Pi_{\rho_{\text {horizon }}}, \Pi_{z_{\text {horizon }}}, \Pi_{\theta_{\text {horizon }}}\right.$ and $\left.\Pi_{t_{\text {horizon }}}\right)$ are satisfied in following commutation relations:

$$
\begin{aligned}
& {\left[\hat{\rho}_{\text {horizon }}, \Pi_{\rho_{\text {horizon }}}\right]=1,\left[\hat{z}_{\text {horizon }}, \Pi_{z_{\text {horizon }}}\right]=1,} \\
& {\left[\hat{\theta}_{\text {horizon }}, \Pi_{\theta_{\text {horizon }}}\right]=1,\left[\hat{t}_{\text {horizon }}, \Pi_{t_{\text {horizon }}}\right]=1}
\end{aligned}
$$

The fundamental definitions are recalled:

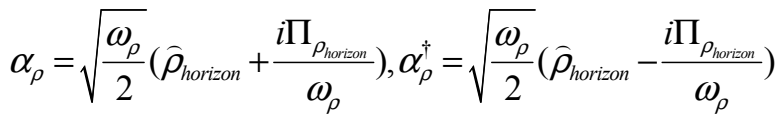

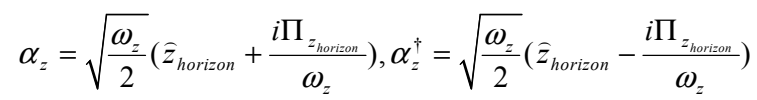

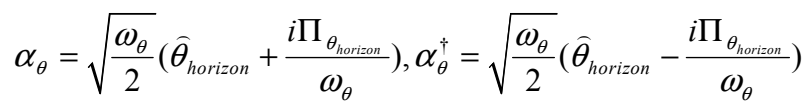

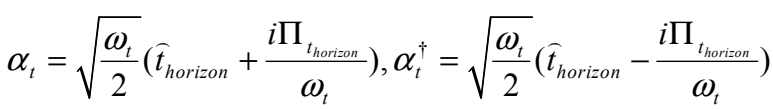

where $\alpha_{\rho}, \alpha_{z}, \alpha_{\theta}, \alpha_{t}$ and $\alpha_{\rho}^{\text {唵 }}, \alpha_{z}, \alpha_{\theta}^{\text {嘹 }}, \alpha_{t}$ are creation and annihilation operators. In agreement with [22-24] we can write the Bogoliubov transformation between

the creation and annihilation operators of expanding and contracting Universe :

$$
\begin{aligned}
& \left(\alpha_{\rho, \exp \text { anding }}-\tanh r_{\rho} \alpha_{\rho, \text { contacting }}^{\dagger}\right) \\
& \left.\mid \text { Universe, } \rho_{\text {horizon }}\right\rangle_{\exp \text { anding } \otimes \text { contracting }}=0 \\
& \tanh r_{\rho}=e^{-2 \pi \omega_{\rho}} \\
& \left(\alpha_{z, \exp \text { anding }}-\tanh r_{z} \alpha_{z, \text { contacting }}^{\dagger}\right) \\
& \left.\mid \text { Universe, } z_{\text {horizon }}\right\rangle_{\exp \text { anding } \otimes \text { contracting }}=0 \\
& \tanh r_{z}=e^{-2 \pi \omega_{z}}
\end{aligned}
$$

$$
\begin{aligned}
& \left(\alpha_{\theta, \exp \text { anding }}-\tanh r_{\theta} \alpha_{\theta, \text { contacting }}^{\dagger}\right) \\
& \left.\mid \text { Universe, } \theta_{\text {horizon }}\right\rangle_{\text {expanding } \otimes \text { contracting }}=0 \\
& \tanh r_{\theta}=e^{-2 \pi \omega_{\theta}} \\
& \left(\alpha_{t, \exp \text { anding }}-\tanh r_{t} \alpha_{t, \text { contacting }}^{\dagger}\right) \\
& \left.\mid \text { Universe, } t_{\text {horizon }}\right\rangle_{\exp \text { anding } \otimes \text { contracting }}=0 \\
& \tanh r_{t}=e^{-2 \pi \omega_{t}}
\end{aligned}
$$

Now, we assume that the Kruskal vacuum

$$
\begin{aligned}
& \left.\mid \text { Universe, } \rho_{\text {horizon }}\right\rangle_{\exp \text { anding } \otimes \text { contracting }}, \\
& \left.\mid \text { Universe, } z_{\text {horizon }}\right\rangle_{\exp \text { anding } \otimes \text { contracting }} \\
& \left., \mid \text { Universe }, \theta_{\text {horizon }}\right\rangle_{\exp \text { anding } \otimes \text { contracting }} \\
& \left., \mid \text { Universe }, t_{\text {horizon }}\right\rangle_{\exp \text { anding } \otimes \text { contracting }}
\end{aligned}
$$

is related to the flat vacuum $|0\rangle_{S}$ by

$$
\begin{aligned}
& \left.\mid \text { Universe, } \rho_{\text {horizon }}\right\rangle_{\exp \text { anding } \otimes \text { contracting }}=F_{\rho}|0\rangle_{S} \\
& \left.\mid \text { Universe, } z_{\text {horizon }}\right\rangle_{\exp \text { anding } \otimes \text { contracting }}=F_{z}|0\rangle_{S} \\
& \left.\mid \text { Universe, } \theta_{\text {horizon }}\right\rangle_{\exp \text { anding } \otimes \text { contracting }}=F_{\theta}|0\rangle_{S} \\
& \left.\mid \text { Universe, } t_{\text {horizon }}\right\rangle_{\exp \text { anding } \otimes \text { contracting }}=F_{t}|0\rangle_{S}
\end{aligned}
$$

where $F$ is some function to be determined later.

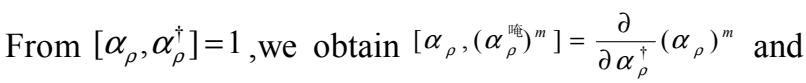
$\left[\alpha_{\rho}, F\right]=\frac{\partial F}{\partial \alpha_{\rho}^{\dagger}}$. Then using equations (12) and (13), we get the following differential equation for $\mathrm{F}$.

$$
\begin{aligned}
& \left(\frac{\partial}{\partial \alpha_{\rho, \exp \text { anding }}^{\dagger}}-\tanh r_{\rho} \alpha_{\rho, \text { contacting }}^{\dagger}\right) F_{\rho}=0 \\
& \left(\frac{\partial}{\partial \alpha_{z, \exp \text { anding }}^{\dagger}}-\tanh r_{z} \alpha_{z, \text { contacting }}^{\dagger}\right) F_{z}=0 \\
& \left(\frac{\partial}{\partial \alpha_{\theta, \exp \text { anding }}^{\dagger}}-\tanh r_{\theta} \alpha_{\theta, \text { contacting }}^{\dagger}\right) F_{\theta}=0 \\
& \left(\frac{\partial}{\partial \alpha_{t, \exp \text { anding }}^{\dagger}}-\tanh r_{t} \alpha_{t, \text { contacting }}^{\dagger}\right) F_{t}=0
\end{aligned}
$$

and the solution is given by

$$
\begin{aligned}
& F_{\rho}=e^{\tanh r_{\rho} \alpha_{\rho, \exp \text { anding }}^{\dagger} \alpha_{\rho, \text { contracting }}^{\dagger}}, \\
& F_{z}=e^{\tanh r_{z} \alpha_{z, \exp \text { anding }}^{\dagger} \alpha_{z, \text { contracting }}^{\dagger}}, \\
& F_{\theta}=e^{\tanh r_{\theta} \alpha_{\theta, \exp \text { anding }}^{\dagger} \alpha_{\theta, \text { contracting }}^{\dagger}}, \\
& F_{t}=e^{\tanh r_{t} \alpha_{t, \exp \text { anding }}^{\dagger} \alpha_{t, \text { contracting }}^{\dagger}}
\end{aligned}
$$


By substituting (15) into (13) and by properly normalizing the state vector, we get

$$
\begin{aligned}
& \left.\mid \text { Universe, } \rho_{\text {horizon }}\right\rangle_{\exp \text { anding } \otimes \text { contracting }}=
\end{aligned}
$$

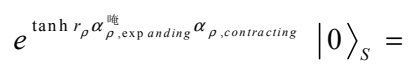

$$
\begin{aligned}
& \frac{1}{\cosh r_{\rho}} \sum_{m} \tanh ^{m} r_{\rho}\left|m_{\rho}\right\rangle_{\exp \text { anding }} \otimes\left|m_{\rho}\right\rangle_{\text {contracting }} \\
& \left.\mid \text { Universe, } z_{\text {horizon }}\right\rangle_{\operatorname{expanding} \otimes \text { contracting }}= \\
& e^{\tanh r_{z} \alpha_{z, \exp \text { anding }}^{\text {撚 }} \alpha_{z, \text { contracting }}}|0\rangle_{S}= \\
& \frac{1}{\cosh r_{z}} \sum_{m} \tanh ^{m} r_{z}\left|m_{z}\right\rangle_{\exp \text { anding }} \otimes\left|m_{z}\right\rangle_{\text {contracting }} \\
& \left.\mid \text { Universe, } \theta_{\text {horizon }}\right\rangle_{\exp \text { anding } \otimes \text { contracting }}= \\
& e^{\tanh r_{\theta} \alpha_{\theta, \exp \text { anding }}^{\text {菂 }} \alpha_{\theta, \text { contracting }}}|0\rangle_{S}= \\
& \frac{1}{\cosh r_{\theta}} \sum_{m} \tanh ^{m} r_{\theta}\left|m_{\theta}\right\rangle_{\exp \text { anding }} \otimes\left|m_{\theta}\right\rangle_{\text {contracting }} \\
& \left.\mid \text { Universe, } t_{\text {horizon }}\right\rangle_{\operatorname{expanding} \otimes \text { contracting }}= \\
& e^{\tanh r_{t} \alpha_{t, \mathrm{exp} \text { anding }} \alpha_{t, c o n t r a c t i n g}}|0\rangle_{S}= \\
& \frac{1}{\cosh r_{t}} \sum_{m} \tanh ^{m} r_{t}\left|m_{t}\right\rangle_{\exp a n d i n g} \otimes\left|m_{t}\right\rangle
\end{aligned}
$$

where $|m\rangle_{\text {expanding }},|m\rangle_{\text {contracting }}$ are orthonormal bases (normal mode solutions) that act on $H_{\exp \text { anding }}$ and $H_{\text {contracting }}$ respectively.By multiplying transversal (physical) degrees of freedom we obtain

$$
\begin{aligned}
& \mid \text { Universe }\rangle_{\text {in } \otimes \text { out }}= \\
& \left.\mid \text { Universe, } \rho_{\text {horizon }}\right\rangle_{\operatorname{expanding} \otimes \text { contracting }} \otimes \\
& \left.\mid \text { Universe, } z_{\text {horizon }}\right\rangle_{\operatorname{expanding} \otimes \text { contracting }} \otimes \\
& \left.\mid \text { Universe, } \theta_{\text {horizon }}\right\rangle_{\operatorname{expanding} \otimes \text { contracting }} \otimes \\
& \left.\mid \text { Universe, } t_{\text {horizon }}\right\rangle_{\operatorname{expanding} \otimes \text { contracting }}
\end{aligned}
$$

We observe that the ground state for oscillating cylindrical Universe is a maximally entangled with two-mode squeezed states on expanding and contracting Hilbert spaces of Universe. We derive the thermal distribution for Universe states as the following:

$$
\begin{aligned}
& N_{\rho}={ }_{\exp a n d i n g \otimes \text { contracting }}\left\langle\text { Universe, } \rho_{\text {horizon }}\right| \alpha_{\rho, \exp a n d i n g}^{\dagger} \\
& \left.\times \alpha_{\rho, \text { contracting }} \mid \text { Universe, } \rho_{\text {horizon }}\right\rangle_{\exp \text { anding } \otimes \text { contracting }} \\
& =\underset{\exp \text { anding }}{ }\left\langle\left. m\right|_{\text {contracting }}\langle m| \frac{1}{\cosh ^{2} r_{\rho}} \alpha_{\rho, \exp a n d i n g}^{\dagger}\right. \\
& \times \alpha_{\rho, \exp a n d i n g} \sum_{n=0}^{\infty} \tanh ^{2 m}\left(r_{\rho}\right)|m\rangle_{\exp a n d i n g}|m\rangle_{\text {contracting }} \\
& ={ }_{\exp \text { anding }}\left\langle m-\left.1\right|_{\text {contracting }}\langle m| \frac{1}{\cosh ^{2} r_{\rho}}\right. \\
& \times \sum_{n=0}^{\infty} \tanh ^{2 m}\left(r_{\rho}\right)(m)|m-1\rangle_{\exp \text { anding }}|m\rangle_{\text {contracting }}=\sinh ^{2} r_{\rho} \\
& N_{z}=\sinh ^{2} r_{z}, N_{\theta}=\sinh ^{2} r_{\theta}, N_{t}=\sinh ^{2} r_{t}
\end{aligned}
$$

Multiplying thermal distributions of horizon states due to expanding and contacting of Universe we have:

$$
\begin{aligned}
& N^{\text {Universe states }} \\
& N_{\rho} N_{z} N_{\theta} N_{t}
\end{aligned}
$$

Equation (19) shows that the probability for producing each state in cyclic Universe depends on different frequencies of Universe in $\rho, z, \theta$ and $t$ directions. To obtain the total production cross section for oscillating cylindical Universe, we need to multiply the Universe surface horizon production cross section by the thermal distribution for Universe states:

$$
\begin{aligned}
& \sigma^{\text {oscillating cylindrical Universe }}= \\
& N^{\text {Universe states }} \sigma^{\text {Universe surface horizon }} \\
& \sigma^{\text {Universesurface horizon }}= \\
& \frac{\left(\rho_{\text {horizon }}^{2} \theta_{\text {horizon }}+z_{\text {horizon }} \rho_{\text {horizon }} \theta_{\text {horizon }}-t_{\text {horizon }}^{2}\right)}{8 \pi^{2} G}
\end{aligned}
$$

This cross section is depended on boundary surface equation and frequencies of oscillating Universe.

\section{The Effect of Universe Oscillation on Higgs Boson Cross Section}

In this section we extend the results of the derivation of Hawking radiation for scalar fields to Higgs boson in cyclic Universe [12,13,22-24]. We show that the ground state for Higgs boson is a maximally entangled two-mode squeezed state on outside and inside Hilbert spaces of surface horizon.

To obtain the Unruh state for Higgs boson,we solve the equation(5) in Kruskal space-time . In Kruskal coordinates the metric of the Universe becomes:

$$
\begin{aligned}
& d s^{2}=-\rho_{\text {horizon }} \frac{e^{-r / \rho_{\text {horizon }}}}{r} d \bar{u} d \bar{v}- \\
& z_{\text {horizon }} \frac{e^{-r / z_{\text {horizon }}}}{r} d \tilde{u} d \tilde{v}-\theta_{\text {horizon }} \frac{e^{-r / \theta_{\text {horizon }}}}{r} d \tilde{u} d \tilde{v}- \\
& t_{\text {horizon }} \frac{e^{-r / t_{\text {horizon }}}}{r} d \tilde{u} d \hat{v}+r^{2} d \Omega^{2}+g_{i j} d x^{i} d x^{j} \\
& \bar{u}=-2 \rho_{\text {horizon }} e^{-u / 2 \rho_{\text {horizon }}}, \bar{v}=-2 \rho_{\text {horizon }} e^{-v / 2 \rho_{\text {horizon }}} \\
& \tilde{u}=-2 z_{\text {horizon }} e^{-u^{\prime} / 2 z_{\text {horizon }}}, \tilde{v}=-2 z_{\text {horizon }} e^{-v^{\prime} / 2 z_{\text {horizon }}}, \\
& \widehat{u}=-2 t_{\text {horizon }} e^{-u^{\prime \prime} / 2 t_{\text {horizon }}}, \widehat{v}=-2 t_{\text {horizon }} e^{-v^{\prime \prime} / 2 t_{\text {horizon }}} \\
& \breve{u}=-2 \theta_{\text {horizon }} e^{-u^{\prime \prime \prime} / 2 \theta_{\text {horizon }}}, \breve{v}=-2 \theta_{\text {horizon }} e^{-v^{\prime \prime \prime} / 2 \theta_{\text {horizon }}}
\end{aligned}
$$




$$
\begin{aligned}
& u=t-\rho^{*}, v=t+\rho^{*}, \\
& \rho^{*}=-\rho-\rho_{\text {horizon }} \ln \left|\rho-\rho_{\text {horizon }}\right| \\
& u^{\prime}=t-z^{*}, v^{\prime}=t+z^{*}, \\
& z^{*}=-z-z_{\text {horizon }} \ln \left|z-z_{\text {horizon }}\right| \\
& u^{\prime \prime}=\rho^{*}+z^{*}+\theta^{*}, v^{\prime \prime}=\rho^{*}-z^{*}+\theta^{*}, \\
& t^{*}=-t-t_{\text {horizon }} \ln \left|t-t_{\text {horizon }}\right| \\
& u^{\prime \prime \prime}=t-\theta^{*}, v^{\prime \prime \prime}=t+\theta^{*}, \\
& \theta^{*}=-\theta-\theta_{\text {horizon }} \ln \left|\theta-\theta_{\text {horizon }}\right|
\end{aligned}
$$

Using this fact that $\bar{v}=0$ on $H^{-}$and $\tilde{v}=0$ on $\tilde{H}^{-}$ $[1,12,13]$ we can estimate the original positive frequency normal mode on these past horizons:

$$
\begin{aligned}
& \text { Higgs boson } \propto\left(e^{-i E_{s} u}\right) \\
& =\left(|\bar{u}| / 2 \rho_{\text {horizon }}\right)^{-i 2 \rho_{\text {horizon }} E_{s}} \\
& = \begin{cases}\left(-\bar{u} / 2 \rho_{\text {horizon }}\right)^{-i 2 \rho_{\text {horizon }} E_{s}} & \text { regionI } \\
\left(\bar{u} / 2 \rho_{\text {horizon }}\right)^{-i 2 \rho_{\text {horizon }} E_{s}} & \text { regionII }\end{cases}
\end{aligned}
$$

Higgs boson $\propto\left(e^{-i E_{s} u^{\prime}}\right)$

$$
\begin{aligned}
& =\left(|\tilde{u}| / 2 z_{\text {horizon }}\right)^{-i 2 z_{\text {horizon }} E_{s}} \\
& = \begin{cases}\left(-\tilde{u} / 2 z_{\text {horizon }}\right)^{-i 2 z_{\text {horizon }} E_{s}} & \text { region } I^{\prime} \\
\left(\tilde{u} / 2 z_{\text {horizon }}\right)^{-i 2 z_{\text {horizon }} E_{s}} & \text { region } I I^{\prime}\end{cases}
\end{aligned}
$$$$
\begin{aligned}
& \text { Higgs boson } \propto\left(e^{-i E_{s} u^{\prime \prime}}\right) \\
& =\left(|\hat{u}| / 2 t_{\text {horizon }}\right)^{-i 2 t_{\text {horizon }} E_{s}} \\
& = \begin{cases}\left(-\hat{u} / 2 t_{\text {horizon }}\right)^{-i 2 t_{\text {horizon }} E_{s}} & \text { region } I^{\prime} \\
\left(\hat{u} / 2 t_{\text {horizon }}\right)^{-i 2 t_{\text {horizon }} E_{s}} & \text { region } I I^{\prime}\end{cases}
\end{aligned}
$$

Higgs boson $\propto\left(e^{-i M_{s} u u^{\prime \prime}}\right)=$

$\left(|\breve{u}| / 2 \theta_{\text {horizon }}\right)^{-i 2 \theta_{\text {horizon }} E_{s}}=$

$$
\begin{cases}\left(-\breve{u} / 2 t_{\text {horizon }}\right)^{-i 2 \theta_{\text {horizon }} E_{s}} & \text { region } I^{\prime} \\ \left(\breve{u} / 2 t_{\text {horizon }}\right)^{-i 2 \theta_{\text {horizon }} E_{s}} & \text { region } I^{\prime}\end{cases}
$$

where Es is the Higgs boson energy .In Eq. (22), we can use the fact that

$$
\begin{aligned}
& (-1)^{-i 2 \rho_{\text {horizon }} E_{s}}=e^{2 \pi \rho_{\text {horizon }} E_{s}},(-1)^{-i 2 z_{\text {horizon }} E_{s}}=e^{2 \pi z_{\text {horizon }} E_{s}} \\
& ,(-1)^{-i 2 t_{\text {horizon }} E_{s}}=e^{2 \pi t_{\text {horizon }} E_{s}},(-1)^{-i 2 \theta_{\text {horizon }} E_{s}}=e^{2 \pi \theta_{\text {horizon }} E_{s}} .
\end{aligned}
$$

Using equation (22) we observe that the states in the horizon satisfy the following condition:

$$
\begin{aligned}
& \left(\text { Higgs boson }_{\text {in }}-\right. \\
& \left.\left.e^{2 \pi \rho_{\text {horizon }} E_{s}} \text { Higgs boson }_{\text {out }}\right) \mid \text { Universe, } \rho_{\text {horizon }}\right\rangle_{\text {in } \otimes \text { out }}=0 \\
& \left(\text { Higgs boson }_{\text {in }}-\right. \\
& \left.\left.e^{2 \pi z_{\text {horizon }} E_{s}} \text { Higgs boson }_{\text {out }}\right) \mid \text { Universe, }, z_{\text {horizon }}\right\rangle_{\text {in } \otimes \text { out }}=0 \\
& \left(\text { Higgs boson }_{\text {in }}-\right. \\
& \left.\left.e^{2 \pi h_{\text {horizon }} E_{s}} \text { Higgs boson }_{\text {out }}\right) \mid \text { Universe, } t_{\text {horizon }}\right\rangle_{\text {in } \otimes \text { out }}=0 \\
& \left(\text { Higgs boson }_{\text {in }}-\right. \\
& \left.\left.e^{2 \pi \theta_{\text {horizon }} E_{s}} \operatorname{Higgs}_{\text {boson }}{ }_{\text {out }}\right) \mid \text { Universe, } \theta_{\text {horizon }}\right\rangle_{\text {in } \otimes \text { out }}=0
\end{aligned}
$$

or equivalently

$$
\begin{aligned}
& \text { (Higgs boson }{ }_{\text {out }}- \\
& \left.\left.\tanh r_{E_{s}, \rho_{\text {horizon }}} \text { Higgs boson }{ }_{\text {in }}\right) \mid \text { Universe, } \rho_{\text {horizon }}\right\rangle_{\text {in } \otimes \text { out }}=0 \\
& \text { (Higgs boson }{ }_{\text {out }} \text { - } \\
& \left.\left.\tanh r_{E_{s}, z_{\text {horizon }}} \text { Higgs boson }{ }_{\text {in }}\right) \mid \text { Universe, } z_{\text {horizon }}\right\rangle_{\text {in } \otimes \text { out }}=0 \\
& \text { (Higgs boson }{ }_{\text {out }} \text { - } \\
& \left.\left.\tanh r_{E_{s}, t_{\text {orrizon }}} \text { Higgs boson }{ }_{\text {in }}\right) \mid \text { Universe, } t_{\text {horizon }}\right\rangle_{\text {in } \otimes \text { out }}=0 \\
& \text { (Higgs boson }{ }_{\text {out }}- \\
& \left.\left.\tanh r_{E_{s}, \theta_{\text {horizon }}} \text { Higgs boson }{ }_{\text {in }}\right) \mid \text { Universe, } \theta_{\text {horizon }}\right\rangle_{\text {in } \otimes \text { out }}=0 \\
& \tanh r_{E_{s}, \rho_{\text {horizion }}}=e^{-2 \pi \rho_{\text {horizion }} E_{s}}, \tanh r_{E_{s}, z_{\text {horizan }}}=e^{-2 \pi z_{\text {horizion }} E_{s}} \\
& \tanh r_{E_{s}, t_{\text {horizon }}}=e^{-2 \pi t_{\text {horizon }} E_{s}}, \tanh r_{E_{s}, \theta_{\text {horizion }}}=e^{-2 \pi \theta_{\text {horizizon }} E_{s}}
\end{aligned}
$$

which actually constitutes a boundary state. Using the expansion in modes for Higgs boson we may write:

$$
\begin{aligned}
& \left.\left(\alpha_{\text {out }}-\tanh r_{E_{s}, \rho_{\text {horizon }}} \alpha_{\text {in }}\right) \mid \text { Universe, } \rho_{\text {horizon }}\right\rangle_{\text {in } \otimes \text { out }}=0 \\
& \left.\left(\tilde{\alpha}_{\text {out }}-\tanh r_{E_{s}, z_{\text {horizon }}} \tilde{\alpha}_{\text {in }}\right) \mid \text { Universe, } z_{\text {horizon }}\right\rangle_{\text {in } \otimes \text { out }}=0 \\
& \left.\left(\widehat{\alpha}_{\text {out }}-\tanh r_{E_{s}, t_{\text {horizon }}} \widehat{\alpha}_{\text {in }}\right) \mid \text { Universe, } t_{\text {horizon }}\right\rangle_{\text {in } \otimes \text { out }}=0 \\
& \left.\left(\breve{\alpha}_{\text {out }}-\tanh r_{E_{s}, t_{\text {horizon }}} \breve{\alpha}_{\text {in }}\right) \mid \text { Universe, }, \theta_{\text {horizon }}\right\rangle_{\text {in } \otimes \text { out }}=0
\end{aligned}
$$

With similar calculations in previous section, we calculate the stationary state of Universe for Higgs boson which is a maximally entangled two-mode state on outside and inside Hilbert spaces:

$$
\begin{aligned}
& \left.\mid \text { Universe, } \rho_{\text {horizon }}\right\rangle_{\text {in } \otimes \text { out }}= \\
& e^{\tanh r_{E_{s}, \rho_{\text {horizon }}}^{\alpha_{\text {out }}^{\text {Wut }} \alpha_{\text {in }}}|0\rangle_{S}=} \\
& \frac{1}{\cosh r_{E_{s}, \rho_{\text {horizon }}}} \sum_{m} \tanh ^{m} r_{E_{s}, \rho_{\text {horizon }}}|m\rangle_{\text {in }} \otimes|m\rangle_{\text {out }}
\end{aligned}
$$




$$
\begin{aligned}
& \left.\mid \text { Universe, } z_{\text {horizon }}\right\rangle_{\text {in } \otimes \text { out }}=
\end{aligned}
$$

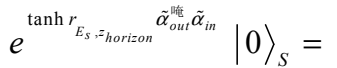

$$
\begin{aligned}
& \frac{1}{\cosh r_{E_{s}, z_{\text {horizon }}}} \sum_{m} \tanh ^{m} r_{E_{s}, z_{\text {horizon }}}|\tilde{m}\rangle_{\text {in }} \otimes|\tilde{m}\rangle_{\text {out }} \\
& \left.\mid \text { Universe, } t_{\text {horizon }}\right\rangle_{\text {in } \otimes \text { out }}=
\end{aligned}
$$

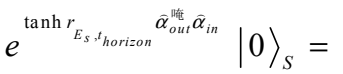

$$
\begin{aligned}
& \frac{1}{\cosh r_{E_{s}, t_{\text {horizon }}}} \sum_{m} \tanh ^{m} r_{E_{s}, t_{\text {horizon }}}|\hat{m}\rangle_{\text {in }} \otimes|\hat{m}\rangle_{\text {out }} \\
& \left.\mid \text { Universe, } \theta_{\text {horizon }}\right\rangle_{\text {in } \otimes_{\text {out }}}= \\
& e^{\tanh r_{E_{s}, \theta_{\text {horizon }}} \hat{\alpha}_{\text {out }}^{\dagger} \hat{\alpha}_{i n}^{\dagger}}|0\rangle_{S}= \\
& \frac{1}{\cosh r_{E_{s}, \theta_{\text {horizon }}}} \sum_{m} \tanh ^{m} r_{E_{s}, \theta_{\text {horizon }}}|\bar{m}\rangle_{\text {in }} \otimes|\bar{m}\rangle_{\text {out }}
\end{aligned}
$$

where $|m\rangle_{\text {in }},|\tilde{m}\rangle_{\text {in }},|\hat{m}\rangle_{\text {in }},|\breve{m}\rangle_{\text {in }}$ and $|m\rangle_{\text {out }},|\tilde{m}\rangle_{\text {out }},|\hat{m}\rangle_{\text {out }},|\tilde{m}\rangle_{\text {out }}$ are orthonormal bases (normal mode solutions) that act on $H_{\text {in }}$ and $H_{\text {out }}$ respectively.By multiplying transversal (physical) degrees of freedom we obtain

$$
\begin{aligned}
& \mid \text { Universe }\rangle_{\text {in } \otimes \text { out }}= \\
& \left.\left.\mid \text { Universe, } \rho_{\text {horizon }}\right\rangle_{\text {in } \otimes \text { out }} \otimes \mid \text { Universe, } z_{\text {horizon }}\right\rangle_{\text {in } \otimes \text { out }} \\
& \left.\left.\otimes \mid \text { Universe, } \theta_{\text {horizon }}\right\rangle_{\text {in } \otimes \text { out }} \otimes \mid \text { Universe, } t_{\text {horizon }}\right\rangle_{\text {in } \otimes \text { out }}
\end{aligned}
$$

We observe that the ground state for Higgs boson near surface horizon is a maximally entangled with two-mode squeezed states on outside and inside Hilbert spaces of Universe. The thermal distribution for Higgs boson is:

$$
\begin{aligned}
& N_{\rho_{\text {horizon }}}= \\
& \text { in out }\left\langle\text { Universe, } \rho_{\text {horizon }}\right| \alpha_{\text {out }}^{\dagger} \\
& \left.\times \alpha_{\text {out }} \mid \text { Universe, } \rho_{\text {horizon }}\right\rangle_{\text {in } \otimes \text { out }} \\
& ={ }_{\text {out }}\left\langle\left. m\right|_{\text {in }}\langle m| \frac{1}{\cosh ^{2} r_{E_{s}, \rho_{\text {horizon }}}} \alpha_{\text {out }}^{\dagger}\right. \\
& \times \alpha_{\text {out }} \sum_{n=0}^{\infty} \tanh ^{2 m}\left(r_{E_{s}, \rho_{\text {horizon }}}\right)|m\rangle_{\text {in }}|m\rangle_{\text {out }} \\
& ={ }_{\text {out }}\left\langle m-\left.1\right|_{\text {in }}\langle m| \frac{1}{\cosh ^{2} r_{E_{s}, \rho_{\text {horizon }}}}\right. \\
& \times \sum_{n=0}^{\infty} \tanh ^{2 m}\left(r_{E_{s}, \rho_{\text {horizon }}}\right)(m)|m\rangle_{\text {in }}|m-1\rangle_{\text {out }} \\
& =\frac{e^{-4 \pi E_{s} \rho_{\text {horizon }}}}{\left(1-e^{\left.-4 \pi E_{s} \rho_{\text {horizon }}\right)}\right.} \\
& \quad N_{z_{\text {horizon }}}=\frac{e^{-4 \pi E_{s} z_{\text {horizon }}}}{\left(1-e^{\left.-4 \pi E_{s} z_{\text {horizon }}\right)}\right.}
\end{aligned}
$$

$$
\begin{aligned}
& N_{t_{\text {horizon }}}=\frac{e^{-4 \pi E_{s} t_{\text {horizon }}}}{\left(1-e^{-4 \pi E_{s} t_{\text {horizon }}}\right)} \\
& N_{\theta_{\text {horizon }}}=\frac{e^{-4 \pi E_{s} \theta_{\text {horizon }}}}{\left(1-e^{-4 \pi E_{s} \theta_{\text {horizon }}}\right)}
\end{aligned}
$$

Multiplying thermal distributions of Higgs boson due to horizon of Universe we have:

$$
\begin{aligned}
& N^{\text {Higgs boson }}= \\
& N_{\rho_{\text {horizon }}} N_{z_{\text {horizon }}} N_{\theta_{\text {horizon }}} N_{t_{\text {horizon }}}
\end{aligned}
$$

Equation (29) shows that different amount of Higgs boson produced with different probabilities inside and outside of Universe. These probabilities are related to Universe horizon and the energy of Higgs boson

$$
P_{n, \omega} \approx \frac{e^{-4 \pi E_{s} \rho_{\text {horizon }}}}{\cosh ^{2} r_{\omega, \rho_{\text {horizon }}}} .
$$

To obtain the total Higgs boson cross section near surface horizon produced due to oscillation of Universe, we need to multiply the oscillating cylindrical Universe production cross section by the thermal distribution of Higgs boson produced near a single horizon.

$$
\begin{aligned}
& \sigma_{\text {Higgs boson }}= \\
& N^{\text {Higgs boson }} N^{\text {Universe states }} \sigma^{\text {Universesurfacehorizon }}
\end{aligned}
$$

Equation(30) shows that in each Universe state,different amount of Higgs boson is produced. In fact, this equation states that the effect of Universe oscillation can observed in Higgs boson production cross section.

\section{The signature of Higgs boson inside the oscillating cylindrical Universe}

If surface horizon is produced inside the Universe, many Higgs bosons produce and decay to to quarks and gluons and this will result in a difference between the observed cosmic ray cross sections and the predicted cross sections. We analyze the signature of Higgs boson inside the oscillating cylindrical Universe.

There are different channels for Higgs decay to QCD matter.The total rate for Higgs boson decaying to gluons at leading order can be derived as $[14,15]$ :

$$
\begin{aligned}
& \Gamma(\text { Higgs } \rightarrow \text { gluons })= \\
& \left(\frac{\alpha_{S}}{\pi}\right)^{2} \frac{\pi}{288 \sqrt{2}}\left[\frac{3}{2 \tau}\left(1+\left(1-\frac{1}{\tau}\right) \arcsin ^{2}(\sqrt{\tau})\right]^{2}\right.
\end{aligned}
$$

in which $\alpha_{S}$ is the strong coupling constant, $\tau=\frac{M_{H}^{2}}{4 M_{t}^{2}}$ is 
the renormalization scale, $\mathrm{MH}$ is the Higgs boson mass and $M_{t}$ is the top quark mass. We assume Higgs bosons annihilate and produce top -anti top quarks with mass $\mathrm{Mt}$, with a subsequent decay to gluons. We denote this gluon production cross section with $\sigma_{p p \rightarrow \text { gluons }}$ and calculate $\sigma_{p p \rightarrow \text { gluons }}$ as following:

$$
\sigma_{\text {gluons }}=\sigma_{\text {Higgs }} \Gamma(\text { Higgs } \rightarrow g g)
$$

We observe that gluon production cross section depends on the second order of strong coupling constant.We also find this cross section increases by increasing the constant.

The rate for Higgs boson decay to bottom-anti bottom quarks can be calculated as $[14,15]$ :

$$
\begin{aligned}
& \Gamma(\text { Higgs } \rightarrow b \bar{b})= \\
& \frac{M_{b}^{2} \alpha_{S}}{6 v^{2} M_{H}^{2}}\left[-4 C_{F} \ln \left(1-\frac{M_{H}^{2}}{Q}\right)\right. \\
& \left.+2 C_{F} \ln \left(\frac{M_{b}^{2}}{M_{H}^{2}}\right)+2 C_{F}\right)
\end{aligned}
$$

Where $v=246 \mathrm{GeV}, C_{F}=\frac{4}{3}, C_{A}=N$

We denote this bottom quark production cross section via process Universe $\rightarrow$ Higgs $\rightarrow b \bar{b}$ by $\sigma_{b \bar{b}}$ with the following calculations:

$$
\sigma_{b \bar{b}}=\sigma_{\text {Higgs }} \Gamma(\text { Higgs } \rightarrow b \bar{b})
$$

When Higgs bosons produce due to oscillation near surface horizon of Universe, they decay to QCD matters .Thus an enhancement of QCD matters can be a signature of Higgs boson inside the Universe.

\section{Summary and Outlook}

In this research we consider the cyclic cylindrical Universes, which experience different oscillating accelerations in each direction. Using the cylindrical coordinates and assuming different accelerations in $\rho, z, \theta$ and t directions, we solve Higgs boson equation in these Universes. After reparameterization, we obtain the Universe metric .Consequently, by considering this metric we derive surface horizon outside of Universe boundary face .Using this metric, we study quantum states of Universe cosmology in cyclic cosmology. We show that the ground state for oscillating cylindrical Universe is a maximally entangled with two-mode squeezed states on expanding and contracting Hilbert spaces of Universe. Next, we obtain the production cross section for each Universe state. We observe that the probability for producing each state in cyclic Universe depends on different oscillating frequencies of Universe in space-time directions. .After that, we consider the effect of Universe oscillation on Higgs boson cross section. To calculate the production cross sec- tion for Higgs boson, we multiply the production cross section for each Universe state by thermal distribution of Higgs boson. We find that in each Universe state,different amount of Higgs boson is produced. Finally, we analyzed the signature of Higgs boson near surface horizon of oscillating cylindrical Universe. When Higgs bosons produce, they decay to quarks and gluons. Thus an enhancement of these partons can be a signature of Higgs boson.

\section{Acknowledgment}

We would like to thank of A. Abitaleb for his lectures about the creation of the Universe that gave us new insight into this subject.

\section{References}

[1] A. Sepehri, S. Shoorvazi, Astrophysics and Space Science,April 2013, Volume 344, Issue 2, pp 521-527

[2] A.Pradhan, A. Rai,S. K.Singh,: Astrophys. Space Sci., Vol. 312, No. 3-4, 261-265 (2007)

[3] A. Pradhan, P. Mathur, Astrophys. Space Sci. Vol. 318, No. 3-4, (20080, 255-261)

[4] A. Pradhan,:Commun. Theor. Phys., Vol. 51, No. 2,367-374 (2009)

[5] A. Pradhan, P.K. Singh, R. K.Jotania,: Czech. J. Phys. Vol. 56, No. 6, 641-660(2006)

[6] Anil Kumar Yadav, Vineet Kumar Yadav, Lallan Yadav : Int. J. Theor. Phys. 48: 568-578,( 2009)

[7] A. Pradhan, M.K. Mishra, A. K. Yadav, Rom. J. Phys. Vol. 54, No. 7-8, (2009), 747-762.

[8] A. Pradhan, S. Agarwal, A. K. Yadav, Rom. J. Phys. Vol. 54, No. 1-2, (2009), 15-28.

[9] A. Pradhan, Journal of Mathematical Physics, Vol. 50, Issue 2, (2009), 022501-022513.

[10] A. Pradhan, K. Jotania, A. Singh, Braz. J. Phys. Vol. 38, No. 1 (2008), 167-177.

[11] I. Chakrabarty, A. Pradhan, N. N. Saste,.: Int. J. Mod. Phys. D, Vol. 10, No. 5, 741-750 (2001)

[12] A. Sepehri, S. Shoorvazi,S. J. Fatemi, S. Doostmohammadi, Astrophysics and Space Science,March 2013, Volume 344, Issue 1, pp 79-86

[13] A. Sepehri, M.E. Zomorrodian, A. Moradi Marjaneh, P. Eslami,S. Shoorvazi, Canadian Journal of Physics, 2012, 90(1): 25-37, 10.1139/p11-134

[14] Mathias Butenschoen, Frank Fugel, Bernd A. Kniehl,Phys.Rev.Lett.98:071602,(2007).

[15] Nikolaos Kidonakis, Phys.Rev.D77:053008,(2008).

[16] Kazuharu Bamba, Kuralay Yesmakhanova, Koblandy Yerzhanov, Ratbay Myrzakulov, accepted for publication in Central European Journal of Physics.( arXiv:1203.3401) 
[17] Jaume de Haro, Accepted for publication in JCAP, arXiv:1207.3621

[18] Kazuharu Bamba, Jaume de Haro, Sergei D. Odintsov, accepted for publication in JCAP, arXiv:1211.2968

[19] Felipe T. Falciano, Nelson Pinto-Neto, E. Sergio Santini, Phys.Rev.D76:083521,2007

[20] Patrick Peter, Nelson Pinto-Neto, Phys.Rev.D78:063506, 2008

[21] Hsin Chen, Fei-Hung Ho, James M. Nester, Chih-Hung Wang, Hwei-Jang Yo, JCAP 0910:027,2009
[22] Mohammad Ebrahim Zomorrodian,Alireza Sepehri, Aliakbar Moradi Marjaneh, Canadian Journal of Physics, 2010, 88(11): 841-849, 10.1139/P10-064

[23] Alireza Sepehri,Somayyeh Shoorvazi ,Mohammad Ebrahim Zomorrodian, Canadian Journal of Physics, 2013, 91(1): 7580, 10.1139/cjp-2012-0163. Doyeol Ahn, Phys. Rev. D74: 084010, (2006).

[24] Alireza Sepehri, Somayyeh Shoorvazi,Mohammad Ebrahim Zomorrodian, Canadian Journal of Physics, 2013, 91(3): 256-259, 10.1139/cjp-2012-0309 